\title{
Survey of Orchidaceae species of Serra das Cabeças, in Parque Estadual da Serra do Brigadeiro, Araponga-MG, Brazil
}

\section{Luísa G Lanaㅜ; Thais F Escobar²; Elis MM Godinho²; Luciano E Peluzio²}

${ }^{1}$ Universidade Federal de Uberlândia (UFU), Uberlândia-MG, Brazil; luisa_g_lana@hotmail.com; ${ }^{2}$ Universidade Federal de Viçosa (UFV), Viçosa-MG, Brazil; thais.escobar@ufv.br; elis.godinho@ufv.br; lpeluzio@ufv.br

\begin{abstract}
Ecology of orchid species was studied, surveyed and characterized on Serra das Cabeças, a subunit of Parque Estadual da Serra do Brigadeiro (PESB), Araponga-MG, Brazil. Data were obtained through monthly visits on all trails of the area, from 2004 to 2014 covering different types of vegetation, collecting some species and identifying them. We observed characteristics such as type of vegetation, habitat where each species was found, intensity of luminosity, altitude and position of plants in relation to watercourses. Obtained results allowed to conclude: 80 species were identified, of which 45 had not been reported for PESB; five for the state of Minas Gerais, and four for Brazilian flora. Concerning habitat, 57.50\% are epiphytic, $1.25 \%$ hemiepiphytic, $2.50 \%$ humic, $15.00 \%$ lithophytic and $23.75 \%$ terrestrial. Considering vegetation, $52.50 \%$ are located in upper montane forest, $1.25 \%$ understorey, $10.00 \%$ slope forest, $8.75 \%$ in transition forest to altitude field, and $27.50 \%$ in altitude field. The survey of Orchidaceae species of present study will allow updating the lists of species present in PESB and of Brazilian flora, recorded in Jardim Botânico in Rio de Janeiro State.
\end{abstract}

Keywords: Orchids, habitat, environmental characteristics, ornamental plants.

\section{RESUMO}

Levantamento de Orchidaceae da Serra das Cabeças do Parque Estadual da Serra do Brigadeiro, Araponga-MG

O estudo trata do levantamento, caracterização e ecologia das espécies de orquídeas da Serra das Cabeças, subunidade do Parque Estadual da Serra do Brigadeiro (PESB), Araponga-MG. Os dados foram obtidos por meio de visitas mensais em todas as trilhas da área, no período de 2004 a 2014 cobrindo os diferentes tipos de vegetação, coletando algumas espécies e identificando-as. Observaram-se características como tipo de vegetação, habitat onde cada espécie foi encontrada, intensidade luminosidade, altitude e posição das plantas em relação a cursos d'água. Os resultados obtidos permitiram concluir: 80 espécies foram identificadas, das quais 45 não haviam sido relatadas para o PESB; cinco para o estado de Minas Gerais e quatro para a flora do Brasil. Quanto ao habitat, 57,50\% são epífitas, 1,25\% hemiepífitas, $2,50 \%$ humícolas, $15,00 \%$ rupícolas e $23,75 \%$ terrestres. Quanto à vegetação $52,50 \%$ encontram-se inseridas em mata altomontano, 1,25\% sub-bosque, 10,00\% mata de encosta, $8,75 \%$ em mata de transição para campo de altitude e $27,50 \%$ em campo de altitude. O levantamento das espécies das Orchidaceae do presente trabalho permitirá a atualização das listas de espécies presentes no PESB e da Flora do Brasil, contidos no registro do Jardim Botânico do Rio de Janeiro.

Palavras-chave: Orquídeas, habitat, características ambientais, plantas ornamentais.

\section{Received on October 18, 2017; accepted on April, 13, 2018}

$\mathrm{T}$ he phytogeographical domain of the studied area is Atlantic Forest, an ecosystem interspersed with Altitude Fields and rocky outcrops, which has as main characteristic the presence of medium and large trees. Both Decree-Law No. 750 of February 10, 1993 and Law No. 11,428 of December 22, 2006 (Atlantic Forest Law) agree with delimitation of the Atlantic Forest domain and definition of phytophysiognomies. Article 2 of Law 11.428/06 presents the following text, very similar to that of Decree-Law no750/93: "For purposes of this law, the following native forest formations and associated ecosystems are considered as members of the Atlantic Forest Domain, with respective delimitations established in a map of the Brazilian Institute of Geography and Statistics (IBGE), according to the regulation: Dense Ombrophilous Forest; Mixed Ombrophilous Forest, also called Araucaria Forest; Open Ombrophilous Forest; Seasonal Semideciduous Forest; and Deciduous Seasonal Forest, as well as mangroves, restinga vegetation, altitude fields, inland swamps, and forest enclaves of the Northeast."
The Atlantic Forest, rich in biodiversity, is composed of large trees which form a microclimate that generates shade and humidity and allows development of several species. It is considered as one of the 25 priority areas for biodiversity conservation (hotspots) in the world (Padilha et al., 2016).

Parque Estatual da Serra do Brigadeiro (PESB) (Serra do Brigadeiro State Park), in Araponga, Minas Gerais, is part of the Atlantic Forest domain and is an ecosystem rich in plant species such as bromeliads, orchids, peroba, ipê, 
cajarana, jequitibá, red oil, and sweet palm. The mist that covers almost all the peaks where the altitude fields are located, provides conditions for the formation of an abundant ecosystem in orchids, ferns, lichens, bromeliads, grasses, shrubs and cacti, among other species (IEF, 2013).

In PESB, the topography is quite rugged, escarpments and massive, presenting great areas of outcrops. Altitude varies from 1,000 to 1,995 m (Engevix apud Silva et al., 2010), being an important influence on the climatic characteristics of the park. Thirteen zones of altitude or mountains are noticed: Pico do Soares, Pico do Boné, Pico do Itajuru, Pico da Ararica, Pico do Cruzeiro, Pico do Grama, Pico do Matipó, Pedra Branca, Cruzeiro, Pedra do Pato, Pedra do Rochedo, Saco do Bode and Serra das Cabeças.

Vegetation in Serra do Brigadeiro is characterized as Semidecidual Seasonal Forest and Dense Ombrophylous Forest (Fernandes et al., 2015; Ribeiro, 2003). We also verified, on the steepest escarpments, a predominant campo rupestre vegetation with unique physiognomic and ecological features, named by Ferri (1980) as altitude fields. This vegetation is well conserved, representing approximately $10 \%$ of PESB area (Paula, 1998), which characterizes landscape of high altitudes, with variable extensions of rocky outcrops, cliffs or rocky peaks (Safford \& Martinelli, 2000). Climate of the region is classified as humid subtropical mesothermic, with an average of three drought months (June, July and August). Average annual temperature is $18^{\circ} \mathrm{C}$, minimum averages below $17^{\circ} \mathrm{C}$ and maximum values below $23^{\circ} \mathrm{C}$, and average rainfall about 1300 mm (Engevix apud Moura et al., 2012).

The Orchidaceae family is originated in Malaysia (Sorgato, 2016), being estimated approximately 25 to 35 thousand species distributed in 850 genera, which present high genetic variability. A large number of hybrids and a huge variety among species, with plants varying from a few centimeters to a few meters in length, different types of habitat, reproduction methods, among other characteristics can demonstrate this great diversity (Cardoso, 2014).

The increasing commercial production of flowers and ornamental plants, among orchids, has been generating income and employment worldwide, increasing environmental benefits and quality of life improvements (Baldotto et al., 2012). Orchids are among the most appreciated ornamental plants in floriculture and of greater commercial value. Thus, their cultivation has increased progressively with the emergence of multiplication and genetic combination techniques, due to their beauty, color diversity, shapes, flower durability, medicinal and culinary properties (Stancato et al., 2001; Pasqual et al., 2011; Suzuki, 2014).

This study was performed to survey and characterize the ecology of the orchid species present in Serra das Cabeças, a subunit of Serra do Brigadeiro State Park, state of Minas Gerais, Brazil, with the records previously proposed.

\section{MATERIAL AND METHODS}

\section{Study area}

The study was carried out from 2004 to 2014, in Parque Estadual da Serra do Brigadeiro (PESB), known before as Serra dos Arrepiados, created on September 27, 1996, through decree No. 38.319 (IEF, 2013). According to Bonfim et al. (2003), the park is located between meridians $40^{\circ} 20^{\prime}$ and $42^{\circ} 40^{\prime} \mathrm{W}$ and parallels $20^{\circ} 33$ and $21^{\circ} 00^{\prime} \mathrm{S}$, 290 kilometers from Belo Horizonte, considering that the 13,984.2708 current hectares are in the Zona da Mata Region, Minas Gerais, in 9 municipalities: Sericita, Araponga, Divino, Ervália, Fervedouro, Miradouro, Muriaé, Pedra Bonita and Rosário da Limeira, at the northern end of Serra do Maciço da Mantiqueira, permeated by the valleys of Carangola, Glória and Doce Rivers (Nunes et al., 2012).

The present study was directed to the subarea Serra das Cabeças (Figure 1), one of the highest points of the park, at $1883 \mathrm{~m}$ altitude, formed by a set of rocky outcrops, eventually called Mamute, Índio or Chinês, and another still not nominated (Castro, 2010). In
Serra das Cabeças, five vegetational formations are noticed, considering slope forest, understorey, upper montane forest, transition forest to altitude field and altitude field. Species of orchids located in this subarea were studied, where some of the essential factors for establishment and development of plants were observed.

\section{Survey of Orchidaceae}

Survey of species was done through monthly visits, in trails which covered all types of vegetation present in Serra das Cabeças (PESB), from 2004 to 2014. Visits were made from the base of the mountains to its top; area was georeferenced and point altitude determined right after location, indicated with aid of a Garmin GPS navigation receiver model Etrex Vista, with precision index, according to manufacturer, of three to five meters in coordinates and fifteen meters in altitude.

\section{Environmental characterization}

The studied orchid species of this region were characterized, the environment where the plants were found, using the following records: identification of the habitat in which the species are inserted; position in relation to the watercourse and average percentage of light incident on the top of the plant, obtained by the comparison between number of luxes incident on the top of plant and the nearest-free environment. Determination was performed using a luxmeter (Instrutherm, model LD-300), which, according to the manufacturer, has a variation index lower than $2 \%$. These are some of the essential factors for establishment and development of plants which allow classifying them as epiphytic, hemiepiphytic, lithophytic, humic or terrestrial, as well as the necessity and adaptability of incident light intensity.

Characterization of Orchidaceae

Characterization and description of species were done through reproductive and vegetative structures of plants (morphological studies), and afterwards photographic record of plant materials (Figures 2, 3 and 4). Parameters obtained in situ associated with measurements and analyses of anatomic and morphologic structures 
were compared with information registered in specific literature, aiming to confirm the identification of species.

Nomenclature used for nominal identification of the species followed parameters determined by Lista de Espécies da Flora do Brasil (The List of Species of the Brazilian Flora) (Barros et al., 2015), established by Jardim Botânico do Rio de Janeiro.

\section{RESULTS AND DISCUSSION}

During the study, the authors found and identified, in Serra das Cabeças, 80 species corresponding to 48 genera of Orchidaceae family (Table 1). Results were compared with the previous survey presented by Leoni \& Tinte (2004), done from May, 2002 to April, 2004, in which the above-mentioned authors concluded that 45 surveyed species are absent and 35 species are present in their study. Species Acianthera langeana (Figure 2A), Acianthera punctatiflora, Acianthera octophrys (Figure 2B), Pabstiella calcarata (Figure 4B), Phymatidium aquinoi. (Figure 4C), of the present survey, are not reported by Barros et al. (2015), for Minas Gerais
State. Species Anathallis gehrtii (Figure 2F), Anathallis pabstii (Figure 2G), Myoxanthus punctatus (Figure 3J), Pleurothallis leucopyramis, of this present survey, were not found in the report according to Barros et al. (2015), for the Brazilian flora.

The authors observed and recorded, in field, the habitat of all orchids found with specimens of the same species living in different habitats, such as Acianthera prolifera (Figure 2C) which was found living as terrestrial and lithophytic, Bifrenaria vitelina which was found as humic and lithophytic, Cyclopogon elegans (Figure 2L) which was found living as terrestrial and humic, Coppensia blanchetii (Figure $2 \mathrm{~K}$ ) living as lithophytic and terrestrial and Promenaea xanthina (figure 4D) living as epiphyte and lithophytic.

According to Zotz (2013) and Joca (2017), approximately $70 \%$ of the species of Orchidaceae family are epiphytes; however, in this study, $57.5 \%$ were recorded, totalizing 46 species from those surveyed in Serra das Cabeças. The authors also identified that the majority of epiphytic orchid species observed were close to groups of bryophytes and lichens.
Such proximity may be associated with better conditions for seed germination and plant maintenance, due to, probably, presence of symbiont microorganisms which would help out in growth process and plant maintenance.

The authors verified a case of primary hemiepiphytes, Vanilla edwallii, which represents $1.25 \%$ of the observed plants. Specimens like this can begin its life cycle as epiphytes (species which germinate on phorophyte) and subsequently establish contact with soil through roots (Geraldino et al., 2010) and this way, they get nutrients for their survival. The humic ones resulted in a total of $2.50 \%$. These species are the ones which live in the forest soil developing their roots among the organic material found around there (Campacci, 2004; Watanabe, 2002).

In Serra das Cabeças, the authors verified $15 \%$ of lithophytic orchids, which live in nature on rocks or hills, normally fixed and associated with decomposed lichens and foliage, accumulated on cracks and recesses in the stones (Campacci, 2004; Watanabe, 2002).

In this same studied area, the authors found $23.75 \%$ of the total orchids living

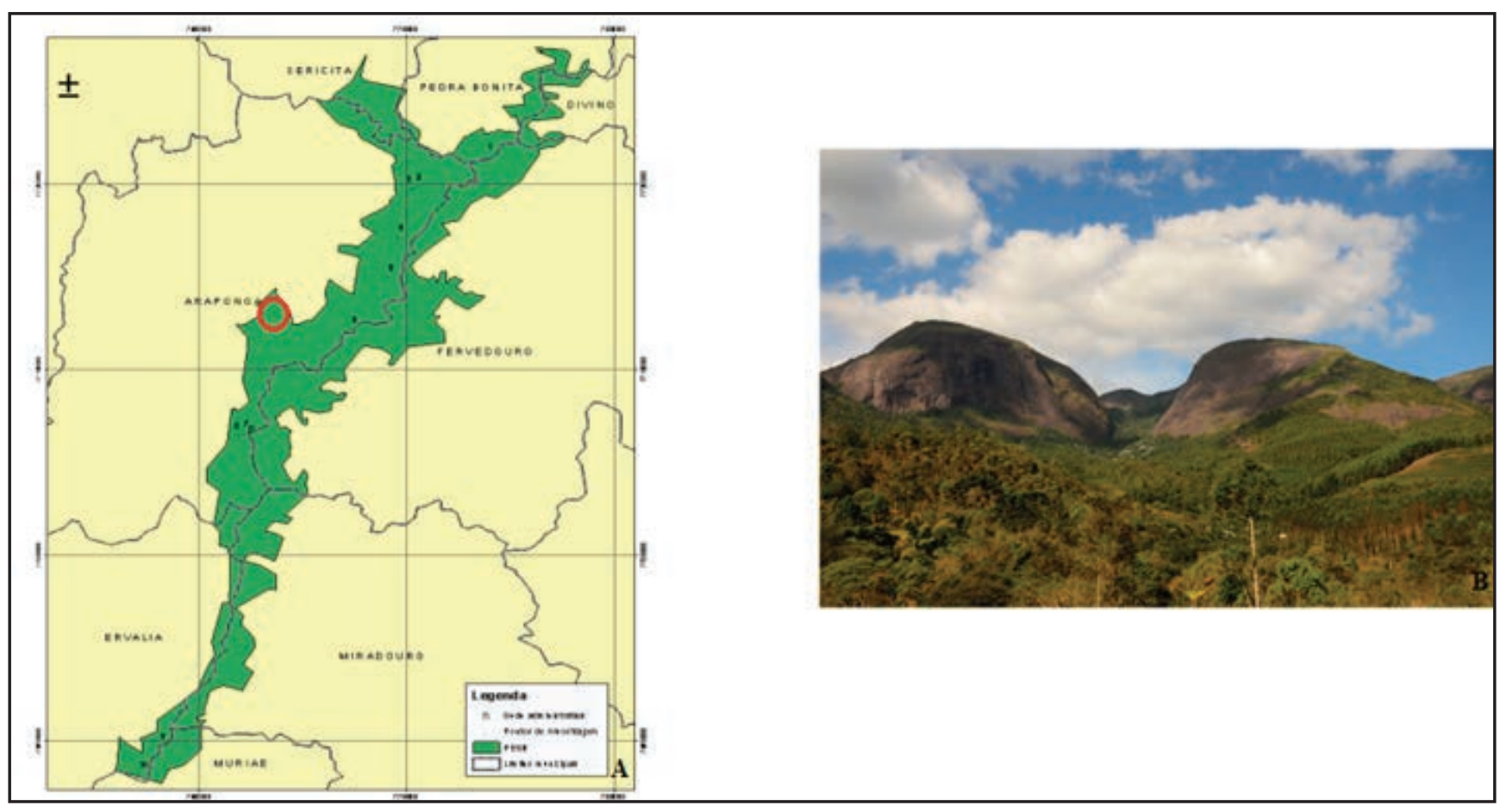

Figure 1. A) Map of the area of Parque Estatual da Serra do Brigadeiro. The red circled area represents Serra das Cabeças (IEF, 2007), B) Picture of Serra das Cabeças in 2013. Uberlandia, UFU, 2004-2014. 
Table 1. Genera and species of Orchidaceae, found from 2004 to 2014, in Serra das Cabeças, Araponga-MG, Brazil, and its relation with the preliminary survey of Parque Estadual da Serra do Brigadeiro (Serra do Brigadeiro State Park, PESB), vegetation, altitude, habitat, watercourse proximity and average percentage of luminosity incident on the plant top comparing the number of luxes on the top of the plant and the nearest-free environment. Uberlandia, UFU, 2004-2014.

\begin{tabular}{|c|c|c|c|c|c|c|}
\hline Genus & Species & $\begin{array}{c}\text { Observation } \\
\text { year }\end{array}$ & $\begin{array}{c}\text { Species of } \\
\text { preliminary } \\
\text { survey (2004) }\end{array}$ & $\begin{array}{c}\text { Vegetation; Altitude (m); } \\
\text { Habitat }\end{array}$ & $\begin{array}{c}\text { Water } \\
\text { course } \\
\text { proximity }\end{array}$ & $\begin{array}{l}\text { Light } \\
(\%)\end{array}$ \\
\hline \multirow{7}{*}{$\begin{array}{l}\text { Acianthera } \\
\text { (Pleurothallis) }\end{array}$} & Acianthera langeana & 2012 & Absent & $\begin{array}{l}\text { Upper montane forest; } 1355 ; \\
\text { epiphyties }\end{array}$ & Yes & 31.8 \\
\hline & Acianthera macropoda & 2004 & Absent & $\begin{array}{c}\text { Upper montane forest; } 1149 ; \\
\text { epiphytes }\end{array}$ & Yes & 45.8 \\
\hline & Acianthera punctatiflora & 2007 & Absent & $\begin{array}{c}\text { Upper montane forest; } 1656 ; \\
\text { epiphytes }\end{array}$ & No & 4.0 \\
\hline & Acianthera octophrys & 2004 & Absent & $\begin{array}{c}\text { Upper montane forest; } 1192 ; \\
\text { epiphytes }\end{array}$ & Yes & 77.8 \\
\hline & Acianthera pubescens & 2008 & Absent & $\begin{array}{c}\text { Upper montane forest; } 1214 ; \\
\text { epiphytes }\end{array}$ & Yes & 71.9 \\
\hline & Acianthera prolifera & 2012 & Present & $\begin{array}{c}\text { Altitude field; } 1670 \text {; terrestrial/ } \\
\text { lithophytes }\end{array}$ & No & 70.0 \\
\hline & Acianthera teres & 2005 & Present & $\begin{array}{l}\text { Upper montane forest; } 1505 \\
\text { lithophytes }\end{array}$ & Yes & 100.0 \\
\hline $\begin{array}{l}\text { Alatiglossum } \\
\text { (Oncidium) }\end{array}$ & Alatiglossum uniflorum & 2010 & Absent & Upper montane forest; 1477 & No & 25.8 \\
\hline \multirow{2}{*}{$\begin{array}{l}\text { Anathallis } \\
\text { (Pleurothallis) }\end{array}$} & Anathallis gehrtii & 2005 & Absent & $\begin{array}{c}\text { Upper montane forest; } 1355 ; \\
\text { epiphytes }\end{array}$ & Yes & 36.0 \\
\hline & Anathallis pabstii & 2004 & Absent & $\begin{array}{l}\text { Upper montane forest; } 1664 ; \\
\text { epiphytes }\end{array}$ & No & 65.3 \\
\hline $\begin{array}{l}\text { Aspidogyne } \\
\text { (Erytrodes) }\end{array}$ & $\begin{array}{l}\text { Aspidogyne } \\
\text { commelinoides }\end{array}$ & 2006 & Present & Upper montane forest; 1306 & Yes & 11.0 \\
\hline $\begin{array}{l}\text { Baptistonia } \\
\text { (Oncidium) }\end{array}$ & Baptistonia truncata & 2009 & Absent & $\begin{array}{l}\text { Upper montane forest; } 1334 \\
\text { epiphytes }\end{array}$ & Yes & 58.0 \\
\hline \multirow{3}{*}{ Bifrenaria } & Bifrenaria tyrianthina & 2010 & Present & $\begin{array}{c}\text { Altitude field; } 1530 \\
\text { lithophytes }\end{array}$ & No & 80.0 \\
\hline & $\begin{array}{c}\text { Bifrenaria tyrianthina } \\
\text { semi-alba }\end{array}$ & 2004 & Absent & $\begin{array}{l}\text { Altitude field; } 1530 ; \\
\text { lithophytes }\end{array}$ & No & 80.0 \\
\hline & Bifrenaria vitelina & 2007 & Absent & $\begin{array}{c}\text { Altitude field; } 1530 \\
\text { lithophytes }\end{array}$ & No & 80.0 \\
\hline $\begin{array}{l}\text { Brasilidium } \\
\text { (Oncidium) }\end{array}$ & Brasilidium crispum & 2011 & Absent & $\begin{array}{l}\text { Upper montane forest; } 1230 ; \\
\text { epiphytes }\end{array}$ & No & 22.6 \\
\hline $\begin{array}{l}\text { Brasiliorchis } \\
\text { (Maxillaria) }\end{array}$ & Brasiliorchis picta & 2006 & Absent & $\begin{array}{l}\text { Altitude field; 1661; } \\
\text { lithophytes }\end{array}$ & No & 18.2 \\
\hline Bulbophyllum & $\begin{array}{l}\text { Bulbophyllum } \\
\text { cantagallense }\end{array}$ & 2009 & Present & Slope forest; 1438; epiphytes & No & 19.64 \\
\hline Campylocentrum & $\begin{array}{c}\text { Campylocentrum } \\
\text { linearifolium }\end{array}$ & 2014 & Absent & $\begin{array}{l}\text { Transition forest/altitude field; } \\
1661 \text {; epiphytes }\end{array}$ & No & 6.8 \\
\hline Centroglossa & Centroglossa macroceras & 2013 & Present & $\begin{array}{l}\text { Upper montane forest; } 1242 \\
\text { epiphytes }\end{array}$ & No & 71.3 \\
\hline Cirrhaea & Cirrhaea dependens & 2010 & Absent & $\begin{array}{l}\text { Upper montane forest; } 1264 ; \\
\text { lithophytes }\end{array}$ & Yes & 25.1 \\
\hline Cleistes & Cleistes gracilis & 2008 & Absent & Altitude field; 1828 ; terrestrial & No & 74.2 \\
\hline \multirow{3}{*}{$\begin{array}{l}\text { Coppensia } \\
\text { (Oncidium) }\end{array}$} & Coppensia barbaceniae & 2005 & Absent & Altitude field; 1828 ; terrestrial & No & 74.2 \\
\hline & Coppensia blanchetii & 2007 & Present & $\begin{array}{c}\text { Altitude field; } 1808 \\
\text { lithophytes }\end{array}$ & No & 82.8 \\
\hline & Coppensia hookeri & 2004 & Present & $\begin{array}{l}\text { Transition forest/altitude field; } \\
\text { 1661; epiphytes }\end{array}$ & No & 3.7 \\
\hline
\end{tabular}

Be continued 
Table 1 continuation

\begin{tabular}{|c|c|c|c|c|c|c|}
\hline Genus & Species & $\begin{array}{c}\text { Observation } \\
\text { year }\end{array}$ & $\begin{array}{c}\text { Species of } \\
\text { preliminary } \\
\text { survey (2004) }\end{array}$ & $\begin{array}{c}\text { Vegetation; Altitude (m); } \\
\text { Habitat }\end{array}$ & $\begin{array}{c}\text { Water } \\
\text { course } \\
\text { proximity }\end{array}$ & $\begin{array}{l}\text { Light } \\
(\%)\end{array}$ \\
\hline \multirow[b]{2}{*}{ Cyclopogon } & Cyclopogon argyrifolius & 2008 & Present & Slope forest; 1190; lithophytes & No & 60.6 \\
\hline & Cyclopogon elegans & 2014 & Absent & $\begin{array}{l}\text { Upper montane forest; } 1244 \\
\text { terrestrial/humic }\end{array}$ & Yes & 32.8 \\
\hline Dichaea & Dichaea bryophyla & 2010 & Present & $\begin{array}{l}\text { Upper montane forest; } 1664 \text {; } \\
\text { epiphytes }\end{array}$ & No & 65.3 \\
\hline \multirow{8}{*}{ Epidendrum } & Epidendrum armeniacum & 2007 & Present & $\begin{array}{l}\text { Upper montane forest; 1334; } \\
\text { epiphytes }\end{array}$ & No & 38.1 \\
\hline & Epidendrum chlorinum & 2007 & Present & Altitude field; 1661; epiphytes & No & 56.1 \\
\hline & $\begin{array}{c}\text { Epidendrum } \\
\text { dendrobioides }\end{array}$ & 2010 & Present & Altitude field; 1661; terrestrial & No & 52.4 \\
\hline & Epidendrum densiflorum & 2008 & Absent & $\begin{array}{l}\text { Upper montane forest; } 1182 \text {; } \\
\text { epiphytes }\end{array}$ & Yes & 71.5 \\
\hline & Epidendrum paranaense & 2006 & Present & $\begin{array}{l}\text { Upper montane forest; } 1553 \text {; } \\
\text { epiphytes }\end{array}$ & No & 50.0 \\
\hline & Epidendrum secundum & 2011 & Present & Altitude field; $1670 ;$ terrestrial & No & 69.6 \\
\hline & Epidendrum xanthinum & 2005 & Present & $\begin{array}{c}\text { Altitude field; } 1835 \\
\text { lithophytes }\end{array}$ & No & 91.2 \\
\hline & Epidendrum sp. & 2005 & Absent & $\begin{array}{c}\text { Upper montane forest; } 1196 \text {; } \\
\text { terrestrial }\end{array}$ & No & 54.8 \\
\hline Eulophia & Eulophia alta & 2014 & Present & Slope forest; 1105; terrestrial & No & 15.2 \\
\hline Eurystyles & Eurystyles actinosophila & 2004 & Present & $\begin{array}{l}\text { Upper montane forest; } 1156 \text {; } \\
\text { epiphytes }\end{array}$ & Yes & 40.6 \\
\hline \multirow{3}{*}{ Gomesa } & Gomesa barker & 2008 & Absent & $\begin{array}{l}\text { Upper montane forest; } 1157 \text {; } \\
\text { epiphytes }\end{array}$ & No & 78.3 \\
\hline & Gomesa glaziovii & 2013 & Absent & Altitude field; 1662; epiphytes & No & 15.1 \\
\hline & Gomesa gomezoides & 2006 & Absent & $\begin{array}{c}\text { Upper montane forest; } 1649 \\
\text { epiphytes }\end{array}$ & Yes & 33.1 \\
\hline Govenia & Govenia utriculata & 2011 & Present & Understorey;1110; terrestrial & No & 55.0 \\
\hline $\begin{array}{l}\text { Grandiphyllum } \\
\text { (Oncidium) }\end{array}$ & $\begin{array}{l}\text { Grandiphyllum } \\
\text { divaricatum }\end{array}$ & 2008 & Present & $\begin{array}{l}\text { Upper montane forest; } 1570 \text {, } \\
\text { epiphytes }\end{array}$ & No & 60.0 \\
\hline Grobya & Grobya amherstiae & 2004 & Absent & $\begin{array}{l}\text { Upper montane forest; } 1210 \\
\text { epiphytes }\end{array}$ & No & 33.8 \\
\hline Habenaria & Habenaria itatiayae & 2009 & Absent & Altitude field; 1662; terrestrial & No & 38.8 \\
\hline $\begin{array}{l}\text { Hadrolaelia } \\
\text { (Sophronitis) }\end{array}$ & Hadrolaelia coccinea & 2009 & Absent & Altitude field; 1783; epiphytes & No & 70.0 \\
\hline $\begin{array}{l}\text { Hoffmanns } \\
\text { eggella }\end{array}$ & $\begin{array}{l}\text { Hoffmanns eggella } \\
\text { cinnabarina }\end{array}$ & 2009 & Present & $\begin{array}{l}\text { Altitude field; } 1655 \\
\text { lithophytes }\end{array}$ & No & 90.0 \\
\hline Isochilus & Isochilus linearis & 2011 & Present & $\begin{array}{c}\text { Upper montane forest; } 1355 ; \\
\text { epiphytes }\end{array}$ & Yes & 36.0 \\
\hline Lankesterella & Lankesterella ceracifolia & 2014 & Absent & $\begin{array}{l}\text { Upper montane forest; } 1664 ; \\
\text { epiphytes }\end{array}$ & No & 9.8 \\
\hline Malaxis & Malaxis excavata & 2014 & Present & $\begin{array}{l}\text { Upper montane forest; } 1650 \text {; } \\
\text { terrestrial }\end{array}$ & No & 50.0 \\
\hline Masdevallia & Masdevallia infracta & 2004 & Present & $\begin{array}{l}\text { Upper montane forest; } 1657 \\
\text { epiphytes }\end{array}$ & No & 57.1 \\
\hline $\begin{array}{l}\text { Mormolyca } \\
\text { (Maxillaria) }\end{array}$ & Mormolyca rufescens & 2008 & Absent & $\begin{array}{l}\text { Upper montane forest; } 1372 \text {; } \\
\text { epiphytes }\end{array}$ & No & 41.3 \\
\hline Myoxanthus & Myoxanthus punctatus & 2005 & Absent & $\begin{array}{c}\text { Upper montane forest; } 1180 \text {; } \\
\text { epiphytes }\end{array}$ & Yes & 82.5 \\
\hline
\end{tabular}

Be continued 
Table 1 continuation

\begin{tabular}{|c|c|c|c|c|c|c|}
\hline Genus & Species & $\begin{array}{c}\text { Observation } \\
\text { year }\end{array}$ & $\begin{array}{c}\text { Species of } \\
\text { preliminary } \\
\text { survey (2004) }\end{array}$ & $\begin{array}{c}\text { Vegetation; Altitude (m); } \\
\text { Habitat }\end{array}$ & $\begin{array}{c}\text { Water } \\
\text { course } \\
\text { proximity }\end{array}$ & $\begin{array}{c}\text { Light } \\
(\%)\end{array}$ \\
\hline \multirow{5}{*}{ Octomeria } & Octomeria anceps & 2006 & Absent & $\begin{array}{c}\text { Upper montane forest; } 1628 \\
\text { epiphytes }\end{array}$ & No & 60.0 \\
\hline & Octomeria concolor & 2006 & Absent & $\begin{array}{l}\text { Transition forest/altitude field; } \\
1661 ; \text { epiphytes }\end{array}$ & No & 20.0 \\
\hline & Octomeria crassifolia & 2013 & Absent & $\begin{array}{c}\text { Upper montane forest; } 1628 \\
\text { epiphytes }\end{array}$ & Yes & 60.0 \\
\hline & Octomeria diaphana & 2011 & Absent & Upper montane forest; 1595 & No & 10.9 \\
\hline & Octomeria linearifolia & 2006 & Absent & Slope forest; 1195; lithophytes & No & 96.6 \\
\hline Oeceoclades & Oeceoclades maculata & 2008 & Absent & Slope forest; 1116; terrestrial & Yes & 100.0 \\
\hline $\begin{array}{l}\text { Pabstiella } \\
\text { (Pleurothallis) }\end{array}$ & Pabstiella calcarata & 2007 & Absent & $\begin{array}{l}\text { Upper montane forest; } 1573 \\
\text { epiphytes }\end{array}$ & No & 40.0 \\
\hline Pelexia & Pelexia laminata & 2009 & Present & $\begin{array}{l}\text { Altitude field; 1505; } \\
\text { lithophytes }\end{array}$ & No & 100.0 \\
\hline Phymatidium & Phymatidium aquinoi & 2010 & Present & $\begin{array}{c}\text { Upper montane forest; } 1573 ; \\
\text { epiphytes }\end{array}$ & No & 40.0 \\
\hline Pleurothallis & $\begin{array}{l}\text { Pleurothallis } \\
\text { leucopyramis }\end{array}$ & 2004 & Absent & Upper montane forest; 1573 & No & 40.0 \\
\hline Polystachya & Polystachya concreta & 2005 & Absent & Slope forest; 1224; epiphytes & No & 0.3 \\
\hline \multirow{3}{*}{ Prescottia } & Prescottia montana & 2008 & Present & $\begin{array}{l}\text { Transition forest/altitude field; } \\
1664 \text {; terrestrial }\end{array}$ & No & 13.1 \\
\hline & Prescottia octopollinica & 2013 & Absent & Altitude field; 1808 ; terrestrial & No & 15.0 \\
\hline & Prescottia stachyoides & 2008 & Present & $\begin{array}{l}\text { Transition forest/altitude field; } \\
1661 ; \text { terrestrial }\end{array}$ & No & 6.8 \\
\hline Promenaea & Promenaea xanthina & 2014 & Present & $\begin{array}{l}\text { Transition forest/altitude field; } \\
1651 ; \text { epiphytes/lithophytes }\end{array}$ & No & 78.7 \\
\hline $\begin{array}{l}\text { Prosthechea } \\
\text { (Encyclia) }\end{array}$ & Prosthechea pygmaea & 2014 & Present & Upper montane forest; 1372 & No & 41.3 \\
\hline $\begin{array}{l}\text { Sacoila } \\
\text { (Stenorrynchos). }\end{array}$ & Sacoila lanceolata & 2006 & Present & Slope forest; 1116; terrestrial & Yes & 100.0 \\
\hline Scaphyglottis & Scaphyglottis modesta & 2011 & Absent & Slope forest; 1180; epiphytes & Yes & 67.3 \\
\hline $\begin{array}{l}\text { Speckclinia } \\
\text { (Pleurothallis) }\end{array}$ & Specklinia marginalis & 2005 & Absent & $\begin{array}{l}\text { Upper montane forest; } 1664 \\
\text { epiphytes }\end{array}$ & No & 55.3 \\
\hline \multirow{2}{*}{ Stelis } & Stelis aprica & 2007 & Absent & $\begin{array}{l}\text { Transition forest/altitude field; } \\
1500 \text {; epiphytes }\end{array}$ & No & 60.1 \\
\hline & Stelis megantha & 2014 & Present & $\begin{array}{l}\text { Upper montane forest; } 1573 \\
\text { epiphytes }\end{array}$ & No & 60.1 \\
\hline Vanilla & Vanilla edwallii & 2006 & $\begin{array}{c}\text { Present } \\
\text { P.................. }\end{array}$ & $\begin{array}{l}\text { Upper montane forest; } 1253 \\
\text { hemiepiphytes }\end{array}$ & No & 75.0 \\
\hline \multirow{5}{*}{ Zygopetalum } & Zygopetalum crinitum & 2013 & Absent & Altitude field; 1828 ; terrestrial & No & 74.0 \\
\hline & Zygopetalum maculatum & 2013 & Absent & Altitude field;1670; terrestrial & No & 67.9 \\
\hline & Zygopetalum maxillare & 2004 & Absent & $\begin{array}{c}\text { Upper montane forest; } 1243 \text {; } \\
\text { epiphytes }\end{array}$ & Yes & 21.4 \\
\hline & $\begin{array}{l}\text { Zygopetalum } \\
\text { pedicellatum }\end{array}$ & 2009 & Absent & Altitude field; 1828 ; terrestrial & No & 74.0 \\
\hline & Zygopetalum triste & 2006 & Present & Altitude field; 1828 ; terrestrial & No & 74.2 \\
\hline
\end{tabular}

light measured (\%) in the environment where each species is located. 

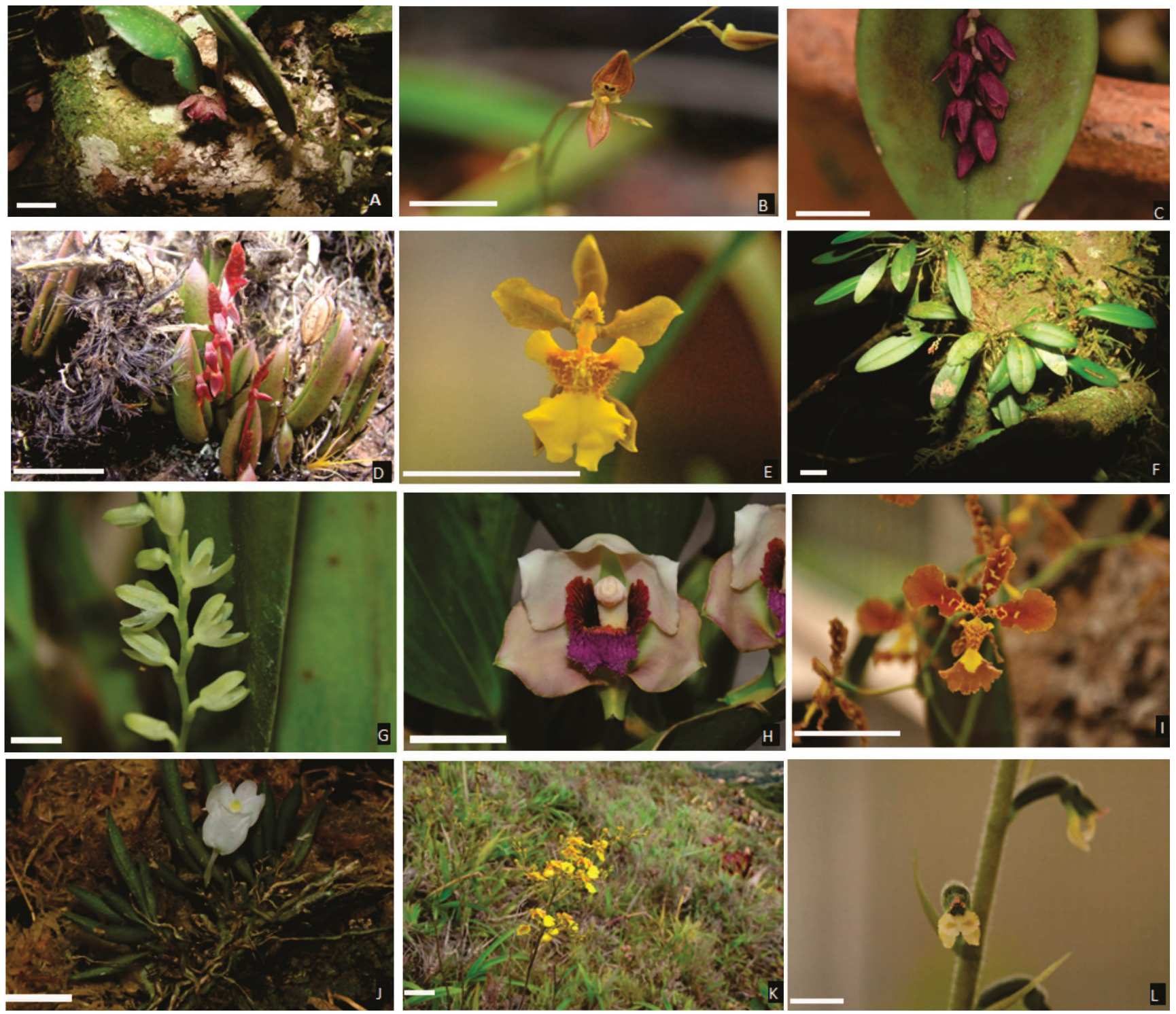

Figure 2. A) Acianthera langeana; B) Acianthera octophrys; C) Acianthera prolifera; D) Acianthera teres; E) Alatiglossum uniflorum; F) Anathallis gehrtii; G) Anathallis pabst; H) Bifrenaria tyrianthina semi-alba I) Brasilidium crispum; J) Centroglossa macroceras; K) Coppensia blanchetii; L) Cyclopogon elegans. White bar represents $1 \mathrm{~cm}$ on the figure. Uberlandia, UFU, 2004-2014.

as terrestrials, which is similar to the statistics reported by Dressler (1981) in which about $25 \%$ of the orchid species are exclusively terrestrials. This result is similar to that of Krahl et al. (2014), who studied the semideciduous forest of South part of Espírito Santo State, finding values of $22.22 \%$ of terrestrial orchids. In this study, the authors recorded a total of 22 species; of all these, twelve were in an altitude-field vegetation, which can be related to the lower vegetative competition index for this kind of vegetation, due to the lowest occurrence of plant species. In this environment vegetation is sparse and plants are adapted to a higher solar irradiation incidence, dominating terrestrial and lithophytic species, for being associated with rocks.

Percentage of species among the types of vegetation was also compared, and the authors concluded that, due to more critical environmental conditions for vegetation development in highlands, the trees existing there are structural, physiognomic and floristically quite different from those located in lower altitudinal floors.

Studying the upper montane forest vegetation, the authors found higher number of species, $52.50 \%$, from which approximately $85 \%$ are epiphytes. Probably, this local presents better conditions for germination and survival of orchid species, factors related to humidity and quantity of necessary nutrients available in soils, besides higher quantity of species acting as support for adaptations of epiphytes of the Orchidaceae family; this seems to be in accordance with Bataghin et al. (2008). These authors reported that, on a local scale, abiotic factors, such as moisture, light and substrate, appear to be directly related to the occupation of the supporting plants by the epiphytes in an upper montane environment. The dossel is commonly uniform and homogeneous, composed of dense and low arboreal vegetation, usually with 

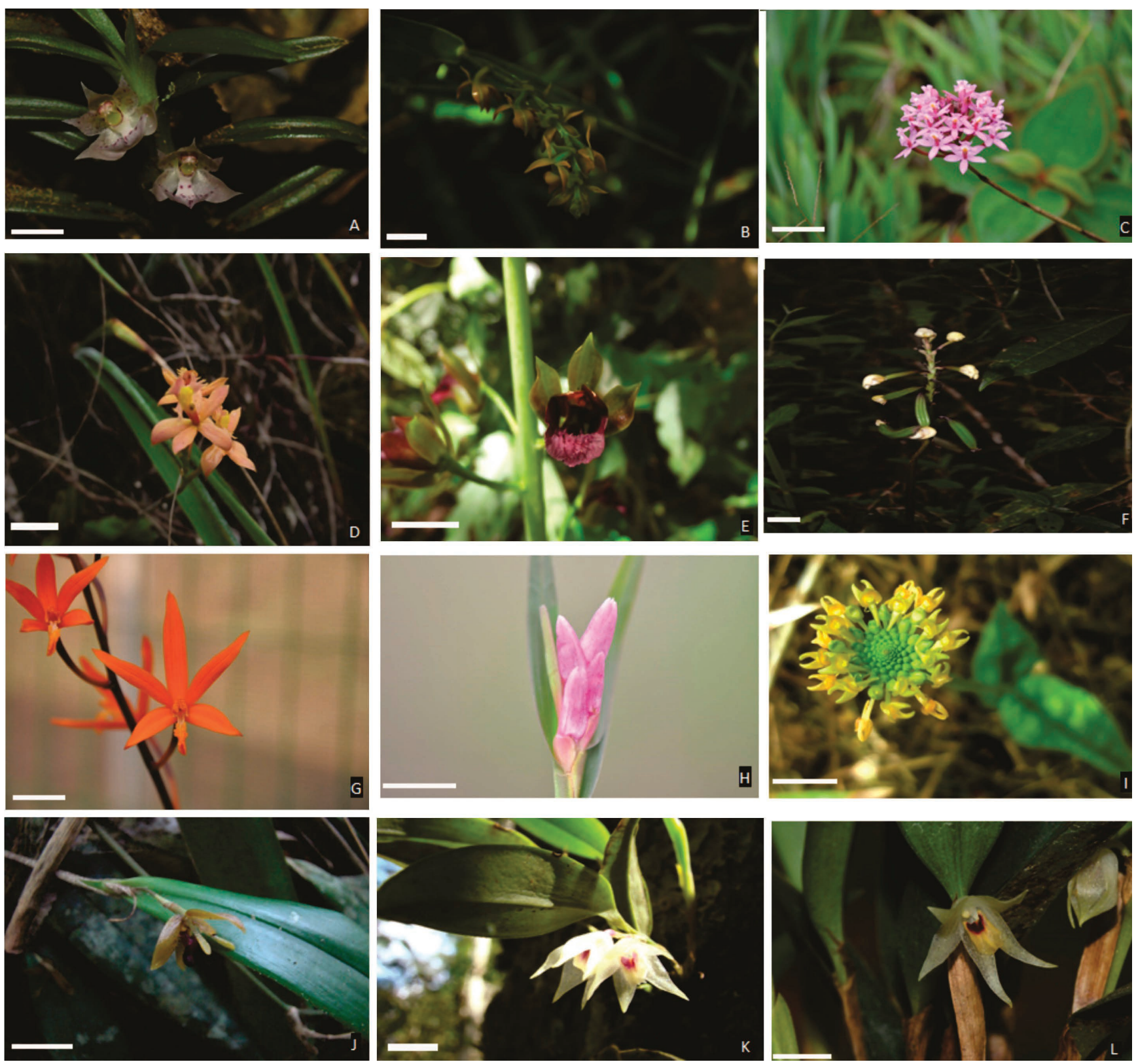

Figure 3. A) Dichaea bryophyla; B) Epidendrum dendrobioides; C) Epidendrum secundum; D) Epidendrum xanthinum; E) Eulophia alta; F) Govenia utriculata; G) Hoffmanns eggella cinnabarina; H) Isochilus linearis; I) Malaxis excavata; J) Myoxanthus punctatus; K) Octomeria anceps; L) Octomeria crassifolia. White bar represents $1 \mathrm{~cm}$ on the figure. Uberlandia, UFU, 2004-2014.

small and sclerophilous leaves and with thin, tortuous and quite branched trunks, totally covered by mosses, lichens and epiphytes (IBGE, 2012; Portes et al., 2001).

According to Leoni \& Tinte (2004), between the slope forest and the altitude field, a transition area can be noticed. In this area, predominate bush species, not exceeding five meters in height, making it easier for the sun's rays to reach the extracts of the understorey, and that the streams of air from the field penetrate into the interior of this type of formation, contributing to dispersion of the seeds belonging to Orchidaceae and Bromeliaceae families which appear in great quantity in this place.

In relation to other species, $1.25 \%$ are found in understorey vegetation, equivalent to species Govenia utriculata (Figure 3F). Possibly, since it is an area presenting a quite short altitude, being closer to human beings, the species which could have been in this environment may have suffered great loss due to the anthropization of the area and the ornamental importance of the orchids.

In slope forest vegetation, the authors recorded $10 \%$ of the total surveyed orchids, adapted to a humid and cold environment: epiphytic, terrestrials and lithophytic, being the species which best adapted to this kind of environment. This slope forest environment presents folic organosol with a thick layer of organic matter, measuring approximately $55 \mathrm{~cm}$, characterizing typical vegetation of semideciduous forests. The microclimatic conditions indicate more humid and colder 

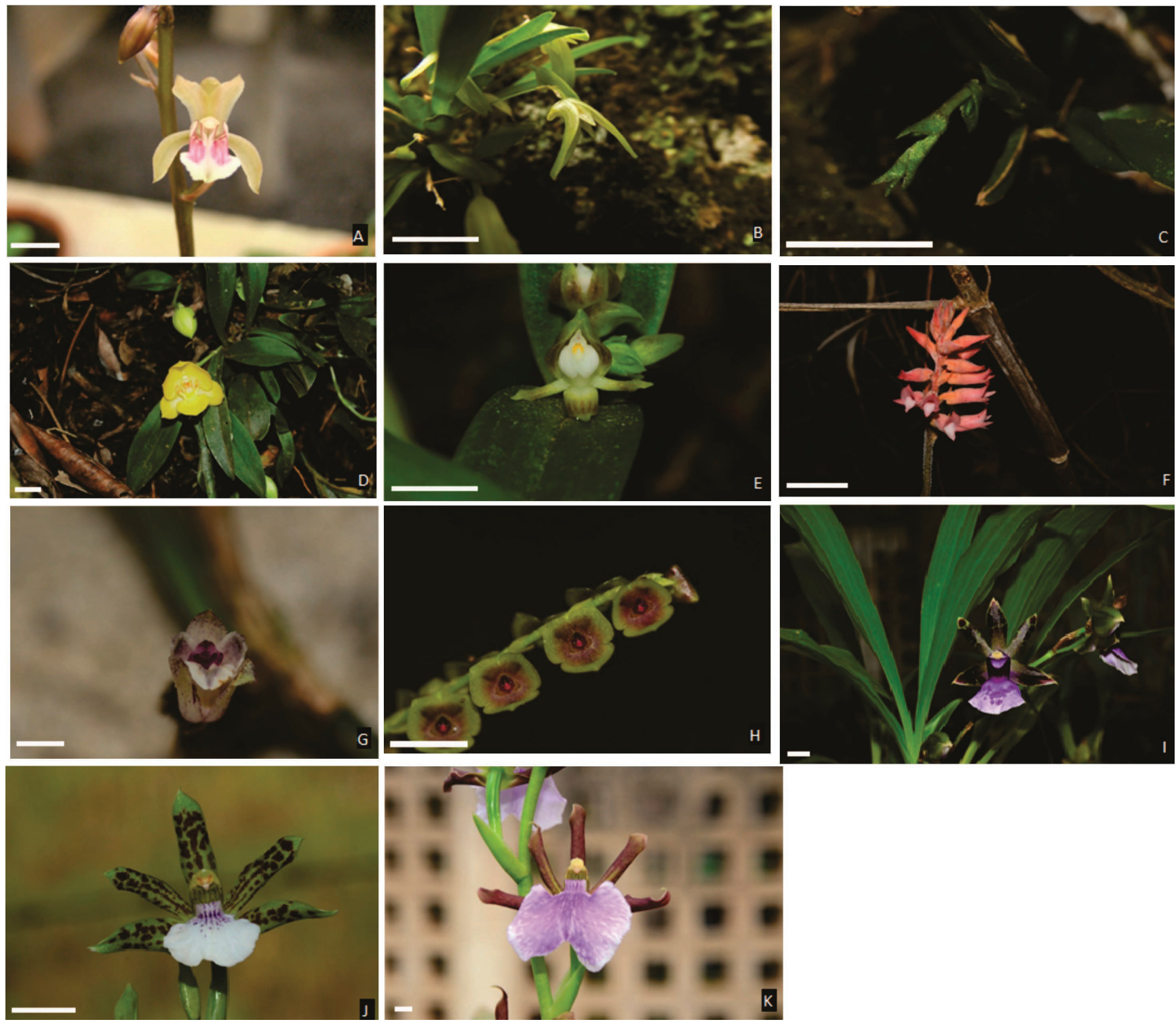

Figure 4. A) Oeceoclades maculata; B) Pabstiella calcarata; C) Phymatidium aquinoi; D) Promenaea xanthina; E) Prosthechea pygmaea; F) Sacoila lanceolata; G) Scaphyglottis modesta; H) Stelis megantha; I) Zygopetalum maxillare; J) Zygopetalum pedicellatum; K) Zygopetalum triste. White bar represents $1 \mathrm{~cm}$ on the figure. Uberlandia, UFU, 2004-2014.

environments and, therefore, at lowerintensity microbial activity, conditioning more conservative character for the environment. Microclimatic conditions show more humid and colder environments; because of this, microbial activity is at lower intensity and more conservative, a determinant character of the environment. That means that this forest area is fed by the decomposition of its own biomass (Moreira et al., 2005).

In forest transition to altitude field, the authors reported that $8.75 \%$ represent rocky outcrop sites, with altitude values above $1600 \mathrm{~m}$, lower vegetation with shrubs and grasses, showing some characteristics of upper montane forest, though. This fact represents a contradiction when characterizing the local vegetation, being called transition vegetation.

Altitude fields, with rocky outcrops, higher than 1,600 $\mathrm{m}$, in general, are in good conditions. The authors reported that $27.50 \%$ of the species found in this research are inserted in this environment. The majority of them are terrestrial, since the predominance of country vegetation in this area favors the terrestrial orchids.

Light is one of the vital elements for all plants, considering that some of them require great luminosity, living in full sun, others would burn if they would be totally exposed to the sun's rays, and still some would only survive in conditions of very low luminosity (Campacci, 2004). The authors observed, with the aid of a luxmeter, that luminosity ranged from 0.3 to $100 \%$, concluding that the orchid species can be much diversified according to the environment they are inserted, being possible to live in very shady environments or in full sun. Thus, the authors could conclude that plants from altitude field receive higher luminosity incidence; however, a plant which is on the top of the tree in a slope forest or upper montane forest will also receive high luminosity incidence. In 
relation to luminosity incidence on the top of the plants, the authors observed that $42.5 \%$ of the species receive less than $50 \%$ irradiation and $57.5 \%$ receive $50 \%$ or more (Table 1 ).

The diverse characteristics of the park, vegetation, location, associated with the hill features, composed of successive crests and valleys, embedded and narrow, provide the emergence of several headwaters of watercourses, being possible to observe some species close to these places or even on them, according to the habitat of the plants, which favors nutrient and water supplies mainly for the epiphytes, which are limited due to their adaptation and the need for a support (Gravendeel et al., 2004). Of the total species, 18 are close to watercourses and most are epiphytes, which demonstrates the importance of being close to water in order to survive, because they are not in contact with water, but in a support near this waterway to get nutrients and water (Table 1).

The survey of Orchidaceae species showed an increase in the reported number of the orchids in the studied area, and allowed characterizing partially the ecology of each species, identifying great differences of adaptation of this group. This knowledge will undoubtedly facilitate the cultivation and management, under controlled conditions, of the mentioned species.

This information will allow updating the lists of species present in PESB and the flora of Minas Gerais and Brazil, with direct reflections on management plan of this Park.

\section{ACKNOWLEDGEMENTS}

The authors thank the administration of Parque Estadual da Serra do Brigadeiro-MG to allow access into the location and for help given during field work.

\section{REFERENCES}

BALDOTTO, LEB; BALDOTTO, MA; SOARES, RR; MARTINEZ, HEP; VENEGAS, VHA. 2012. Adventitious rooting in cuttings of croton and hibiscus in response to indolbutyric acid and humic acid, Viçosa, Minas Gerais. Ceres 59: 476-483.

BARROS, F; VINHOS, F; RODRIGUES, VT; BARBERENA, FFVA; FRAGA, CN; PESSOA, EM; FORSTER, W; MENINI NETO, NL; FURTADO, SG; NARDY, C; AZEVEDO, CO; GUIMARÃES, LRS. 2015. Orchidaceae in Lista de Espécies da Flora do Brasil. Jardim Botânico do Rio de Janeiro. Available in <http://floradobrasil.jbrj.gov. br/jabot/floradobrasil/FB11230 $>$. Accessed July 7, 2015.

BATAGHIN, FA; FIORI, A; TOPPA, RH. 2008. Efeito de borda sobre epífitos vasculares em floresta ombrófila mista, Rio Grande do Sul, Brasil. O Mundo da Saúde São Paulo 32: 329-338.

BONFIM, VR; RIBEIRO, GA; SILVA, E; BRAGA, GM. 2003. Diagnóstico do uso do fogo no entorno do Parque Estadual da Serra do Brigadeiro (PESB), MG. Revista Árvore 27: 87-94.

CAMPACCI, MA. 2004. História e cultivo das orquideas. Caderno do orquidofilo, $3^{\mathrm{a}} \mathrm{ed}$. Taubaté: Edit. Brasil orquídeas. 48p.

CARDOSO, JC. 2014. Levantamento de espécies da família Orchidaceae em Botucatu: potencial ornamental e cultivo. Horticultura Brasileira 32: 7-13.

CASTRO, H. 2010 Parque Estadual da Serra do Brigadeiro. Clube dos Aventureiros, Rio de Janeiro. Available in $<$ http//www. clubedosaventureiros.com/>. Accessed March 14, 2013.

DRESSLER, RL. 1981. The orchids: natural history and classification. Cambridge: Harvard University Press. 317p.

FERNANDES, JM; GARCIA, FCP; SIQUEIRA, LC; MAROTTA, CPB; CARDOSO, IM. 2015. Riqueza e a similaridade de Leguminosae em sistemas agroflorestais cafeeiros em Araponga, Minas Gerais. Revista Brasileira de Agroecologia 10: 75-86.

FERRI, MG. 1980. Vegetação Brasileira. São Paulo: Editora USP. 157p.

GRAVENDEEL, B; SMITHSON,, A; SLIK, FJ; SCHUITEMAN, A. 2004. Epiphytism and pollinator specialization: drivers for orchid diversity? Philosophical Transactions of the Royal Society 359: 1523-1535.

GERALDINO, HCL; CAXAMBÚ, MG; SOUZA, DC. 2010. Composição florística e estrutura da comunidade de epífitas vasculares em uma área de ecótono em Campo Mourão, PR, Brasil. Acta Botânica Brasilica 24: 469-482.

IBGE. 2012. Manual técnico da vegetação brasileira. Fundação Instituto Brasileiro de Geografia e Estatística. Rio de Janeiro: IBGE. 66-134.

IEF - INSTITUTO ESTADUAL DE FLORESTAS. 2007. Plano de manejo do Parque Estadual da Serra do Brigadeiro. Available in <http:// www.ief.mg.gov.br/images/stories/Plano de_Manejo/serra_do_brigadeiro/pesb $\% 2 \overline{0}$ encarte $\% 201 \% 20-\% 20$ diagnstico $\% 20$ do $\% 20$ parque.pdf $>$. Accessed December 21, 2017.

IEF - INSTITUTO ESTADUAL DE FLORESTAS. 2013. Parque Estadual da Serra do Brigadeiro. Available in <http://www.ief.mg.gov.br/ component/content/197?task=view $>$. Accessed February 20, 2015.

JOCA, TAC; OLIVEIRA, DC; ZOTZ, G; WINKLER, U; MOREIRA, ASFP. 2017. The velamen of epiphytic orchids: variation in structure and correlations with nutrient absorption. Flora 230: 66-74.

KRAHL, AH; COGO, AJD; VALSKO, JJ. 2014. Orchidaceae em um fragmento de floresta semidecídua de encosta na região sul do Estado do Espírito Santo, Sudeste do Brasil. Hoehnea 41: 247-268.

LEONI LS; TINTE VA. 2004. Flora do Parque Estadual da Serra do Brigadeiro Minas Gerais, Brasil: caracterização da vegetação e lista preliminar das espécies. Carangola: Fundação Faculdade de Filosofia, Ciências e Letras de Carangola. 91p.

MOURA, MR; MOTTA, AP; FERNANDES, VD; FEIO, RN. 2012. Herpetofauna da Serra do Brigadeiro, um remanescente de Mata Atlântica em Minas Gerais, Sudeste do Brasil. Biota Neotropica 12: 209-235.

MOREIRA, GF; OLIVEIRA, FS; FRANÇA, MM; SOUZA, E; FARIA, ALL. 2005. Estratificação ambiental numa topossequência no Parque da Serra do Brigadeiro-MG. Caderno de Geografia 15: 78-95.

NUNES, AV; LESSA, G; SCOSS, LM. 2012. Composição e abundância relativa dos mamíferos terrestres de médio e grande porte do Parque Estadual da Serra do Brigadeiro, Minas Gerais, Brasil. Biotemas 25: 205-216.

PADILHA, PT; ELIAS, GA; AZEREDE, TEV; COLARES, R; ANTUNES, AR; CITADINIZANETTE, V. 2016. Comunidade de bromélias epifíticas em um fragmento florestal urbano no sul de Santa Catarina, Brasil. Revista Tecnologia e Ambiente 22: 82-100.

PASQUAL, M; SOARES, JDR; RODRIGUES, FA; ARAUJO, AG; SANTOS, RR. 2011. Influência da qualidade de luz e silício no crescimento in vitro de orquídeas nativas e híbridas. Horticultura Brasileira 29: 324-329.

PAULA, CC. 1998. Florística da familia Bromeliaceae no Parque Estadual da Serra do Brigadeiro, Minas Gerais, Brasil. Rio Claro: UNESP. 238p. (Tese doutorado).

PORTES, MCGO; GALVÃO, F; KOEHLER, A. 2001. Caracterização florística e estrutural de uma floresta ombrófila densa altomontana do morro do Anhangava, Quatro Barras-PR. Floresta 31: 1-10.

RIBEIRO, CAN. 2003. Florística e fitossociologia de um trecho de Floresta Atlântica de Altitude na Fazenda da Neblina, Parque Estadual da Serra do Brigadeiro, Minas Gerais.Viçosa: UFV. 52p. (Dissertaçãomestrado).

SAFFORD, HD; MARTINELLI, G. 2000. Southeast Brazil. In: POREMBSKI, S; BARTHLOTT, W (eds). Inselbergs: biotic diversity of isolated rock outcrops in Tropical and Temperate regions. Alemanha: Springer Verlag. p.339-389.

SILVA, IV; MEIRA, RMSA; AZEVEDO, AA. 2010. Anatomia de raízes de espécies de Orchidaceae do Parque Estadual da Serra do Brigadeiro, Minas Gerais. Hoehnea 37: 147-161.

SORGATO, JC. 2016. Protocolo de germinação 
em meios assimbióticos para Dendrobium nobile Lindl. e Dendrobium phalaenopsis Fitzg. Dourados: UFGD. 54p. (Tese doutorado).

STANCATO, GC; BEMELMANS, PF; VEGRO, CLR. 2001. Produção de mudas de orquídeas a partir de sementes in vitro e sua viabilidade econômica: estudo de caso. Revista Brasileira de Horticultura Ornamental 7: 25-33.

SUZUKI, RM. 2014. Breve análise sobre o comércio exterior de orquídeas no Brasil. In: $21^{\text {a }}$ Reunião Anual do Instituto de Botânica, 2014. São Paulo. Anais... São Paulo: Instituto de Botânica. P.1-4.

WATANABE. 2002. Orquídeas: manual de cultivo. São Paulo: AOSP. 296p.

ZOTZ, G. 2013. The systematic distribution of vascular epiphytes-a critical update. Botanical Journal of the Linnean Society. 171: 453-481. 